\title{
Altered acetylcholinesterase as a resistance mechanism in the brown planthopper (Homoptera: Delphacidae), Nilaparvata lugens Stål
}

\author{
Jai-Ki Yoo, ${ }^{*}$ Si-Woo Lee, Yong-Joon Ahn, ${ }^{1}$ Toru Nagata ${ }^{2}$ and Toshio Shono ${ }^{3}$ \\ National Institute of Agricultural Science and Technology, RDA, Suwon 441-707, Korea \\ ${ }^{1}$ Division of Applied Biology and Chemistry and the Research Center for New Bio-Materials in Agriculture, Seoul National Uni- \\ versity, Suwon 441-744, Korea \\ ${ }^{2}$ Laboratory of Applied Entomology and Zoology, Faculty of Agriculture, Ibaraki University, Ibaraki 300-0393, Japan \\ ${ }^{3}$ Laboratory of Applied Zoology, Institute of Agriculture and Forestry, University of Tsukuba, Tsukuba, Ibaraki 305-8572, Japan
}

(Received 8 June 2001; Accepted 3 October 2001)

\begin{abstract}
Highly resistant strains of the brown planthopper were obtained after 30 generations of laboratory selection by carbofuran or fenobucarb. Topical $\mathrm{LD}_{50}$ for fenobucarb increased 93-101-times and topical $\mathrm{LD}_{50}$ for carbofuran increased 51-68-times on selection by either carbofuran or fenobucarb, while the $\mathrm{LD}_{50}$ for diazinon increased only 6-7-times by the same selections. Sensitivity of AChE to carbofuran or fenobucarb was reduced remarkably in vitro in the resistant strains while sensitivity to diazoxon changed only slightly. Insensitive AChE was considered to be the major resistance mechanism of the carbamate-resistant strains of the brown planthopper.
\end{abstract}

Key words: Nilaparvata lugens, acetylcholinesterase, resistance, carbofuran, fenobucarb

\section{INTRODUCTION}

The brown planthopper (BPH), Nilaparvata lugens Stål, is one of the most devastating insect pests of rice in Asia. Unless management of this pest is operated properly from the early growth stage of the crop, this insect causes serious yield loss directly by feeding, and indirectly by transmission of the grassy stunt virus disease in the tropics.

BPH migrates to Korea every year from foreign breeding sources such as mainland China (Kishimoto, 1971; Lee and Park, 1977; Ma, 1993). This insect undergoes two or three generations in paddy fields during the summer season in Korea. Control of BPH is entirely dependent on insecticide application. Although insecticides were once effective for controlling $\mathrm{BPH}$, their continuous use has reduced the biological regulatory function of natural enemies, resulting in resurgence (Chiu, 1979; Heinrichs, 1979), and also led to development of insecticide resistance in this insect (Nagata et al., 1979; Chung et al., 1982; Ahn et al., 1993). To minimize these problems, it is necessary to reduce the amount of insecticides used for BPH control, and retard resistance development.

Monitoring of resistance development in $\mathrm{BPH}$ has been conducted extensively in Korea (Yoo et al., 1997). The mechanism of resistance, however, has not been studied well in BPH. This paper deals with the resistance mechanism, especially the altered acetylcholinesterase (AChE) in BPH. It is well known that the altered $\mathrm{AChE}$ is one of the main resistance mechanism in many insect pests. Insensitivity of AChE observed in organophosphate- or carbamate-resistant insects has been well documented on various arthropod species (Hama, 1983; Devonshire and Moores, 1984; Oppenoorth, 1985; Hemingway et al., 1986; Fournier and Mutero, 1994). In this paper, as a non-metabolic mechanism of resistance, insensitivity of AChE to carbofuran, fenobucarb and diazoxon in the laboratory-selected strains of BPH was studied.

\section{MATERIALS AND METHODS}

Insects. Three strains of BPH were used. The susceptible strain (referred to as $\mathrm{S}$ ) has been maintained for 19 years in the laboratory of the National Institute of Agricultural Science and Technology without any exposure to insecticides. Strains resistant to carbofuran and fenobucarb were obtained after selection of S strain by spraying a water solu-

\footnotetext{
* To whom correspondence should be addressed.
} 
tion of $10 \%$ carbofuran wettable powder (referred to as Rc-30) or $50 \%$ fenobucarb emulsifiable concentrate (referred to as Rf-30) for 30 generations under a selection pressure of 30-70\% mortality. The selection was performed on 3-4th stadium nymphs by the spray method using a Potter's spray tower (Burkard, UK). Planthoppers surviving $24 \mathrm{~h}$ after treatment were reared on rice seedlings (var. Chucheong; 7-10 d after germination) in an acrylic cages $(26 \times 30 \times 20 \mathrm{~cm})$ under long-day condition of LD $16: 8,26 \pm 1{ }^{\circ} \mathrm{C}$ and $65 \pm 5 \%$ relative humidity $(\mathrm{RH})$ to facilitate production of offsprings.

Bioassay. Insecticide susceptibilities were determined by topical application. A droplet of acetone $(0.05 \mu \mathrm{l})$ containing the appropriate concentration of insecticide was applied to individual female adults and the $24 \mathrm{~h}$ mortality was applied to a probit analysis to determine $\mathrm{LD}_{50}$ values.

Chemicals. Carbofuran (2,3 dihydro-2,2-dimethylbenzofuran-7-yl methyl carbamate, $75.8 \%$ purity), fenobucarb (2-sec-butyl phenyl methyl carbamate, $98.2 \%$ purity) and diazinon (O,O-diethyl $O$-2-isopropyl-6-methylpyrimidin-4-yl phosphorothioate, $98.2 \%$ purity) were obtained from Kyungnong Chemical (Seoul, Republic of Korea) and diazoxon $(O, O$-diethyl $O$-2-isopropyl-6-methyl pyrimidin-4-yl phosphate, $98.7 \%$ purity) was kindly supplied by Sungbo Chemical (Seoul). Acetylthiocholine iodide, DTNB, and eserine salicylate were purchased from Sigma. All other chemicals used were of reagent grade.

Acetylcholinesterase (AChE) inhibition. The in vitro inhibition of $\mathrm{AChE}$ by carbofuran, fenobucarb and diazoxon was determined by the method of Ellman et al. (1961), using acetylthiocholine (ATCh) iodide as a substrate. Twenty four- to fived-old female adults were homogenized in $20 \mathrm{ml}$ of ice-cold $0.1 \mathrm{M}$ phosphate buffer ( $\mathrm{pH}$ 7.4). After filtering through cheese cloth, the homogenate was centrifuged at $10,000 \times g$ for $20 \mathrm{~min}$. The supernatant was directly used as the AChE enzyme source. A series of concentrations of carbofuran, fenobucarb and diazoxon in acetone $(10 \mu \mathrm{l})$ were placed in the test tubes and the solvent (acetone) was blown dried with nitrogen gas. Incubation mixture consisting of $1 \mu \mathrm{l}$ of enzyme solution (equivalent to 1 female $/ \mathrm{ml}), 2 \mathrm{ml} 0.1 \mathrm{M}$ phosphate buffer, $100 \mu 10.1 \mathrm{~mm}$ DTNB in buffer was added and samples were placed at $30^{\circ} \mathrm{C}$ in a shaking water bath for $10 \mathrm{~min}$. Then $20 \mu \mathrm{l}$ of $0.075 \mathrm{~mm}$ acetyl- choline iodide was added to the mixture. After incubation for $20 \mathrm{~min}$ at $30^{\circ} \mathrm{C}$, the reaction was stopped by adding $0.2 \mathrm{ml}$ of $5 \mathrm{~mm}$ eserine salicylate. The AChE activity was spectrophotometrically measured at $412 \mathrm{~nm}$. The $\mathrm{I}_{50}$ value which reduced $50 \%$ of the AChE activity was determined by probit analysis (Raymond, 1985).

\section{RESULTS}

After continuous selection for 30 generations by either carbofuran or fenobucarb, a high level of resistance to the insecticides developed in the selected strains (Table 1). Carbofuran selection increased the $\mathrm{LD}_{50}$ for carbofuran and fenobucarb by 68 times and 101 times, respectively. Fenobucarb selection also increased the resistance level by 51 times and 93 times against carbofuran and fenobucarb, respectively, while the $\mathrm{LD}_{50}$ for diazinon increased only 6-7-times in either of the two selected strains.

The in vitro $\mathrm{AChE}$ inhibition of $\mathrm{S}$ strain and resistant strains (Rc-30 and Rf-30) at various concentrations of carbofuran, fenobucarb and diazoxon are shown in Figs. 1-3. AChE of Rc-30 and Rf-30 exhibited a marked reduction in their sensitivity to the two carbamate insecticides compared with that of S strain. Insensitivity ratios of the Rc-30 ( $\mathrm{I}_{50}$ values of Rc-30/I $\mathrm{I}_{50}$ value of $\mathrm{S}$ strain) to carbofuran and fenobucarb were 19.2 and 21.8, and those of the Rf-30 to carbofuran and fenobucarb were 107.7 and 39.4, respectively. But AChE of the two carba-

Table 1. Toxicity of carbofuran, fenobucarb and diazinon to resistant, Rc-30 and Rf-30 strains

\begin{tabular}{llrcrrr}
\hline \multirow{2}{*}{ Strains } & Insecticides & \multicolumn{2}{c}{ Resistant } & & \multicolumn{2}{c}{$\mathrm{S}$} \\
\cline { 3 - 4 } & & $\begin{array}{c}\mathrm{LD}_{50} \\
(\mu \mathrm{g} / \mathrm{g})\end{array}$ & $\begin{array}{c}\mathrm{CL}^{\mathrm{a}} \\
(\mu \mathrm{g} / \mathrm{g})\end{array}$ & & $\begin{array}{c}\mathrm{LD}_{50} \\
(\mu \mathrm{g} / \mathrm{g})\end{array}$ & $\mathrm{RR}^{\mathrm{b}}$ \\
\hline \multirow{2}{*}{$\mathrm{Rc}-30$} & Carbofuran & 20.3 & $13.4-91.1$ & & 0.3 & 68 \\
& Fenobucarb & 166.9 & $119.2-217.5$ & 1.7 & 101 \\
& Diazinon & 23.5 & $17.7-30.5$ & 3.9 & 6 \\
Rf-30 & Carbofuran & 15.4 & $9.1-23.7$ & 0.3 & 51 \\
& Fenobucarb & 155.1 & $107.4-390.7$ & 1.7 & 93 \\
& Diazinon & 27.9 & $19.7-36.9$ & 3.9 & 7
\end{tabular}

a $95 \%$ confidence limit.

${ }^{\mathrm{b}}$ Resistance ratio $\left(\mathrm{LD}_{50}\right.$ of resistant strain/ $\mathrm{LD}_{50}$ of susceptible strain). 


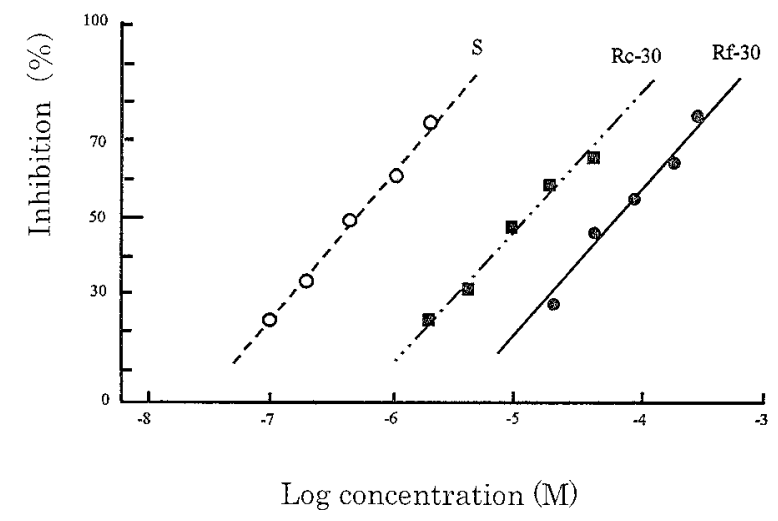

Fig. 1. Inhibition of acetylcholinesterase by carbofuran in resistant and susceptible female adult $\mathrm{BPH}$.

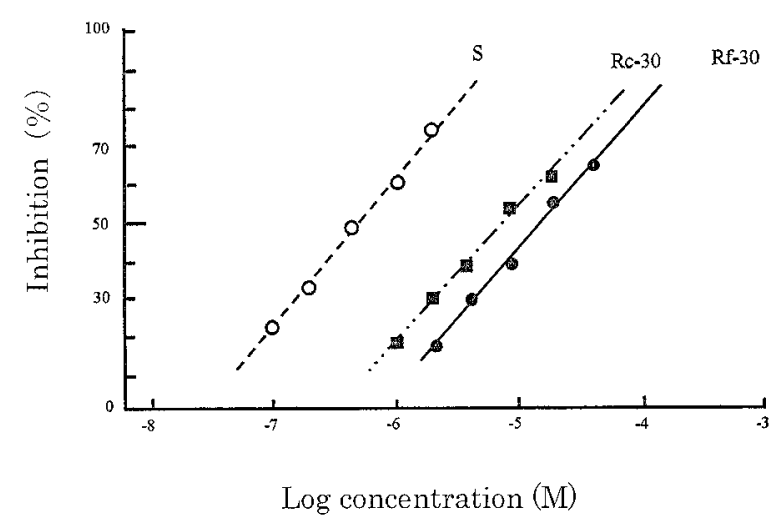

Fig. 2. Inhibition of acetylcholinesterase by fenobucarb in resistant and susceptible female adult $\mathrm{BPH}$.

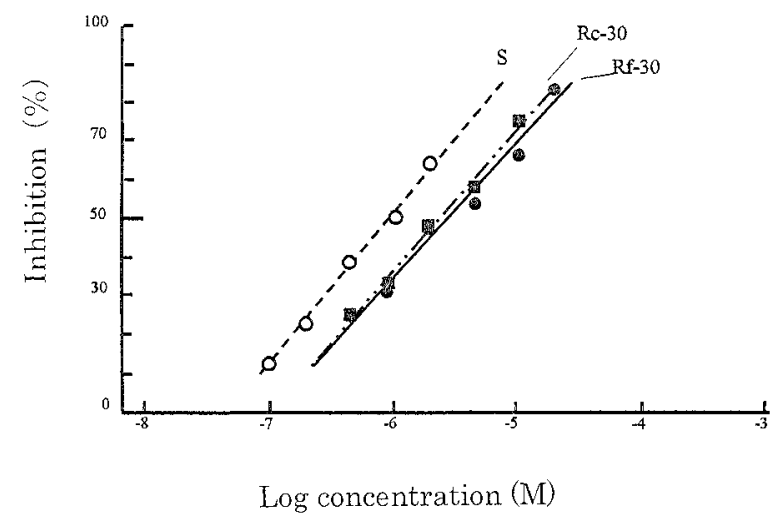

Fig. 3. Inhibition of acetylcholinesterase by diazoxon in resistant and susceptible female adult $\mathrm{BPH}$.

mate resistant strains was only slightly reduced in sensitivity to diazoxon; the insensitivity ratios of the Rc-30 and Rf-30 were 3.0 and 3.6, respectively (Table 2).
Table 2. Inhibition of acetylcholinesterase in the resistant, Rc-30, Rf-30 and the susceptible (S) female adult BPH by carbofuran, fenobucarb and diazoxon

\begin{tabular}{|c|c|c|c|}
\hline \multirow{2}{*}{ Strains } & \multicolumn{3}{|c|}{$\mathrm{I}_{50}{ }^{\mathrm{a}}(\mathrm{mM})$} \\
\hline & Carbofuran & Fenobucarb & Diazoxon \\
\hline Rc-30 & $1.5 \times 10^{-2}(19.2)^{b}$ & $7.2 \times 10^{-3}(21.8)$ & $2.8 \times 10^{-3}(3.0)$ \\
\hline Rf-30 & $8.4 \times 10^{-2}(107.7)$ & $1.3 \times 10^{-2}(39.4)$ & $3.3 \times 10^{-3}(3.6)$ \\
\hline $\mathrm{S}$ & $7.8 \times 10^{-4}$ & $3.3 \times 10^{-4}$ & $9.2 \times 10^{-4}$ \\
\hline
\end{tabular}

${ }^{\text {a }}$ Concentrations inhibiting 50\% of acetylcholinesterase.

${ }^{\mathrm{b}}$ Figures in the parenthesis indicate insensitivity ratios $\left(\mathrm{I}_{50}\right.$ values of resistant strain $/ \mathrm{I}_{50}$ value of the $\mathrm{S}$ strain).

\section{DISCUSSION}

Development of resistance to the carbamates by laboratory selection on BPH is generally slow as compared with those to organophosphates and pyrethroids. Chung et al. (1982) selected BPH with MIPC for 16 generations and observed a 34-fold increase in $\mathrm{LC}_{50}$ values. In this study we obtained highly resistant strains by selection for 30 generations, which showed a 93-102-fold resistance ratio for fenobucarb and 51-68-fold for carbofuran. Comparison of $\mathrm{LD}_{50}$ values between Rc-30 and Rf30 showed the presence of remarkable and nearly equal levels of cross-resistance between carbofuran and fenobucarb, but no cross-resistance to diazinon was observed (Table 1). This finding suggests that the resistance mechanism of both strains to carbofuran and fenobucarb might be exactly the same, but it does not work on diazinon though diazinon belongs to the same category of AChE inhibitors as the carbamates, carbofuran and fenobucarb.

The largest topical $\mathrm{LD}_{50}$ ever reported for a field population of $\mathrm{BPH}$ is ca. $50 \mu \mathrm{g} / \mathrm{g}$ for fenobucarb and ca. $10 \mu \mathrm{g} / \mathrm{g}$ for carbofuran (Nagata, 1999). Our laboratory-selected strains have a much larger $\mathrm{LD}_{50}$ than the field strains and these strains facilitated analysis of the resistance mechanism.

Insensitive AChE acts as one of the major mechanisms of resistance to organophosphates and carbamate insecticides in many insects (Russell, 1980; Hama, 1983). In this study, the resistant strains showed a remarkable reduction in the sensitivity of AChE to the in vitro inhibition by carbofuran and fenobucarb, suggesting that the major mechanism of resistance to carbofuran and fenobucarb in $\mathrm{BPH}$ is undoubtedly insensitivity of AChE to the carba- 
mates. The altered AChE has been verified in $\mathrm{BPH}$ as a common mechanism of resistance to organophosphates and carbamates (Hama and Hosoda, 1983; Miyata et al., 1983), although this mechanism was first found in the two-spotted spider mite (Smissaert, 1964). Chung and Sun (1983) reported that AChE insensitivity was not associated with malathion resistance in $\mathrm{BPH}$, but $\mathrm{AChE}$ in the MIPC-resistant strain was 15.7-fold less sensitive to MIPC, indicating that $\mathrm{AChE}$ insensitivity was one of the main resistance factors to MIPC.

Insensitivity of $\mathrm{AChE}$ contributes differently to resistance to diazinon and carbamates in the two carbamate-resistant strains of $\mathrm{BPH}$ though the mode of action is common among all three insecticides. Significant AChE insensitivity was observed to carbamate insecticides in Rc-30 and Rf-30 strains of $\mathrm{BPH}$, whereas $\mathrm{AChE}$ of the resistant BPH showed only slight insensitivity to diazoxon. Both resistant strains, therefore, exhibited no cross resistance to diazinon.

While Rc-30 showed almost equal insensitivity ratios to both carbofuran and fenobucarb, Rf-30 showed a higher insensitivity ratio to those insecticides than Rc-30, especially to carbofuran. The resistant Rf-30 might have some supplementary mechanism of resistance in addition to AChE insensitivity. It is interesting that the pattern of resistance development varies between insecticides which have the same mode of action as anti-AChE insecticides.

\section{REFERENCES}

Ahn, Y. J., H. M. Park, J. K. Yoo and J. O. Lee (1993) Resistance mechanism of insecticides in the brown planthopper (Nilaparvata lugens Stål) and strategies of its control in the fields. In Establishment, Spread, and Management of the Rice Water Weevil and Migratory Rice Insect Pests in East Asia. Agricultural Sciences Institute, Suwon, Korea, pp. 275-291.

Chiu, S. C. (1979) Biological control of the brown planthopper. In Brown Planthopper: Threat to Rice Production in Asia. IRRI, Los Baňos, Philippines, pp. 335-355.

Chung, T. C. and C. N. Sun (1983) Malathion and MIPC resistance in Nilaparvata lugens (Homoptera: Delphacidae). J. Econ. Entomol. 76: 1-5.

Chung, T. C., C. N. Sun and C. Y. Hung (1982) Resistance of Nilaparvata lugens to six insecticides in Taiwan. J. Econ. Entomol. 75: 199-200.

Devonshire, A. L. and G. D. Moores (1984) Characterization of insecticide-insensitive acetylcholinesterase: microcomputer-based analysis of enzyme inhibition in homogenates of individual house fly (Musca domestica) heads. Pestic. Biochem. Physiol. 21: 341-348.

Ellman, G. L., K. D. Courtney, V. Andres, Jr. and R. M. Featherstone (1961) A new and rapid colorimetric determination of acetylcholinesterase activity. Biochem. Pharmacol. 7: 88-95.

Fournier, D. and A. Mutero (1994) Modification of acetylcholinesterase as a mechanism of resistance to insecticides. Comp. Biochem. Physiol. 108C: 19-31.

Hama, H. (1983) Resistance to insecticides due to reduced sensitivity of AChE. In Pest Resistance to Pesticides (G. P. Georghiou and T. Saito eds.). Plenum Press, New York, pp. 299-331.

Hama, H. and A. Hosoda (1983) High aliesterase activity and low acetylcholinestease sensitivity involved in organophosphorus and carbamate resistance of the brown planthopper, Nilaparvata lugens Stål (Homoptera: Delphacidae). Appl. Entomol. Zool. 18: 475-485.

Heinrichs, E. A. (1979) Chemical control of the brown planthopper, Nilaparvata lugens (Stål). In Threat to Rice Production in Asia. IRRI, Los Baňos, Philippines, pp. 145167.

Hemingway, J., C. Smith, K. G. Jayawardena and P. R. J. Herath (1986) Field and laboratory detection of the altered acetylcholinesterase resistance genes which confer organophosphate and carbamate resistance in mosquitoes (Diptera: Culicidae). Bull. Entomol. Res. 76: 559-565.

Kishimoto, R. (1971) Long distance migration of planthoppers, Sogatella furcifera and Nilaparvata lugens. In Proceedings, Rice Insects. Trop. Agric. Res. Cent. Jpn. Minist. Agric. Forest., Tokyo, pp. 201-206.

Lee, J. O. and J. S. Park (1977) Biology and control of the brown planthopper (Nilaparvata lugens $\mathrm{S}$ ) in Korea. In The Rice Brown Planthopper. FFTC, ASPAC, Taipei, pp. 199-213.

Ma, C. (1993) Occurrence of the rice planthoppers in northeastern China and their northward migration. In International Workshop: Establishment, Spread, and Management of the Rice Water Weevil and Migratory Rice Insect Pests in East Asia. National Agriculture Research Center, Tsukuba, Japan, pp. 307-322.

Miyata, T., T. Saito and K. Ozaki (1983) In vitro degradation of malathion by organophosphate resistant and susceptible strains of brown planthopper, Nilaparvata lugens Stål. J. Pesticide Sci. 8: 27-31.

Nagata, T. (1999) Monitoring of insecticide resistance of the brown planthopper and the white backed planthopper in Asia. In Intercountry Forecasting System and Management for Brown Planthopper in East Asia. RDA and FAO, Suwon, Korea, pp. 167-185.

Nagata, T., T. Masuda and S. Moriya (1979) Development of resistance in the brown planthopper, Nilaparvata lugens Stål (Hemiptera: Delphacidae). Appl. Entomol. Zool. 14: 264-269.

Oppenoorth, F. J. (1985) Biochemistry and genetics of insecticide resistance. In Comprehensive Insect Physiology, Biochemistry and Pharmacology: Insect Control, Vol. 12 (G. A. Kerkut and L. I. Gilbert eds.). Pergamon, Oxford, pp. 731-733.

Raymond, M. (1985) Presentation d'un programme d'analyse 
log-probit pour microordimateut. Ent. Med. Parasitol. 22: $117-121$

Russell, A. (1980) Cholinesterase inhibitors. In Introduction to Biochemical Toxicology (E. Hodgson and F. E. Guthrie eds.). Blackwell Science Pub., Oxford, pp. 193-223.

Smissaert, H. R. (1964) Cholinesterase inhibition in spider mites susceptible and resistant to organophosphate. Sci- ence 143: 129-131.

Yoo, J. K., H. M. Park, B. R. Choi, S. G. Lee and Y. J. Ahn (1997) Resistance monitoring and cross-resistance of brown planthopper (Homoptera: Delphacidae), Nilaparvata lugens Stål in Korea. Korean J. Pestic. Sci. 1: 41-47. 